\title{
Synthesis, Molecular Docking and Biological Evaluation of Some Novel Heterocyclic Derivatives
}

\author{
Eman M. Flefel, W. A. Tantawy, A. A. Fayed, Hayam H. \\ Sayed and M. Khedr" \\ Department of Photochemistry, National Research Centre and \\ *Pharmaceutical Chemistry Department, Faculty of Pharmacy, \\ Helwan University, Helwan, Egypt.
}

\begin{abstract}
A SERIES of heterocyclic derivatives 2-15 including pyrimidinone and pyrimidine thione rings were synthesized by using chalcones $1 \mathrm{a}-\mathrm{c}$ as starting materials. Treatment of compound $1 \mathrm{a}$ with hydrogen peroxide afforded the corresponding epoxide 2, which afford thioxopyrimidine derivative 3 upon the reaction with thiourea. The latter compound was treated with halo-derivatives to give the corresponding $\mathrm{N}$-substituted thioxopyrimidine derivatives 4 and 5 . Moreover, compounds 6-10 were prepared by the treatment of compound $1 \mathrm{~b}$ with acetyl acetone, hydrazine hydrate, urea, thiourea or thiosemicarbazide, respectively. Also, compound 10 was condensed with p-chlorobenzaldehyde and chloroethanol to give 11 and 12, respectively. The alkylation of $9 \mathrm{a}$ with methyl iodide or ethylchloroacetate accessing the $S$-substituted pyrimidine derivatives 13 and 14, respectively was carried out. Also, compound 9a was reacted with p-methoxybenzaldehyde and bromoacetic acid to afford the corresponding thiazolopyrimidine 15. Characterization and structural elucidation of the products was realized based on chemical and spectral analyses. Furthermore, a molecular docking study was performed in order to predict the anticancer activity of these compounds against Tyrosine Kinase enzyme hoping to find any promising affinity that could be of potential anticancer activity. Also, the biological evaluation of some prepared compounds has been assessed and some of them revealed promising antioxidant activity.
\end{abstract}

Keywords: Pyrimidinethione, Acyclic nucleosides, Molecular docking and Antioxidant activity.

Heterocyclic compounds having nitrogen and sulfur in their skeleton are among the most fascinating compounds due to their diverse biological activities. These compounds play an important role in medicinal chemistry ${ }^{(1)}$, serving as key templates to the development of numerous important therapeutic agents ${ }^{(2.3)}$. Pyrimidinethione derivatives have found application in a wide range of medical applications because of their diverse biological activities, such as anti-HIV-1 
agents $^{(2,3)}$, anticancer ${ }^{(4,5)}$, antiviral ${ }^{(6,7)}$, antimicrobial ${ }^{(8-11)}$, anti-inflammatory ${ }^{(12,13)}$ and potential antitubercular agents ${ }^{(14)}$. Thiazolopyrimidine has good antiinflammatory, analgestic anticonvulsant and antiparkinsonian ${ }^{(15)}$, anticancer ${ }^{(16)}$, and binding affinity to the CRH-R1 receptor, thus showing promise as a new class of potential anxiolytics and/or antidepressants ${ }^{(17)}$. Recently, new synthetic pyrimidinethione and pyrazole derivatives have showed a considerable activity as antioxidants $^{(18)}$. Moreover, many scientists had focused on the natural antioxidants such as ascorbic acid and $\alpha$-tocopherol rather than synthetic antioxidants such as butylated hydroxyanisole (BHA) and butylated hydroxytoluene (BHT), to scavenge the oxygenated reactive species (ORS) which cause several health problems including cancer, heart diseases and possibly, the process of aging itself $^{(19)}$.

The current work aims to synthesize new compounds of anticipated antioxidant and anticancer activity.

In doing so, we employed the molecular docking technique to predict the affinities of our synthesized compounds toward tyrosine kinases (TK) inhibition $^{(20)}$ and hence their predicted anticancer effect. Recent advances have implicated the role of tyrosine kinases in the physiology of cancer. Though their activity is tightly regulated in normal cells, they may acquire transforming functions due to mutation(s), over expression leading to malignancy. The activation of cancer cells can be blocked by selective tyrosine kinase inhibitors and thus considered to be a promising target for anticancer drug discovery. Tyrosine kinases are important mediators of the signal transduction process ${ }^{(21)}$, leading to cell proliferation, differentiation, migration, metabolism and programmed cell death. Tyrosine kinases are a family of enzymes, which catalyzes phosphorylation of select tyrosine residues in target proteins, using $\mathrm{ATP}^{(21)}$. They are implicated in several steps of neoplastic development and progression. Tyrosine kinase signaling pathways normally prevent deregulated proliferation or contribute to sensitivity towards apoptotic stimuli. These signaling pathways are often genetically or epigenetically altered in cancer cells to impart a selection advantage to the cancer cells.

\section{Results and Discussion}

2,4-Dichloroacetophenone was reacted with aromatic and heterocyclic aldehydes in ethanolic sodium hydroxide solution to produce 3-(substituted)-1(2,4-dichloro-phenyl)-propen-1-ones (1a-c) as starting materials. Compound 1a was reacted with hydrogen peroxide in alkaline medium to give $(2,4-$ dichlorophenyl)-3-(4-methoxyphenyl)oxiran-2-yl)methanone (2), which was condensed with thiourea in alcoholic potassium hydroxide to afford the corresponding thioxo-pyrimidinone (3). When compound 3 was reacted with 2,3,4,6-tetra- $O$-acetyl- $\alpha$-D-glucopyranosyl bromide or alkyl chloride in alkaline medium gave the corresponding 4-(4-methoxy-phenyl)-6-(2,4-dichlorophenyl)1,3 -di- $(2,3,4,6$ - tetra- $O$ - acetyl- $\beta$-D- glucopyranosyl)-2- thioxotetra- hydro-

Egypt. J. Chem. 55, No.4 (2012) 
pyrimidin -5 - one (4) and compounds 5a,b, respectively (Scheme 1).

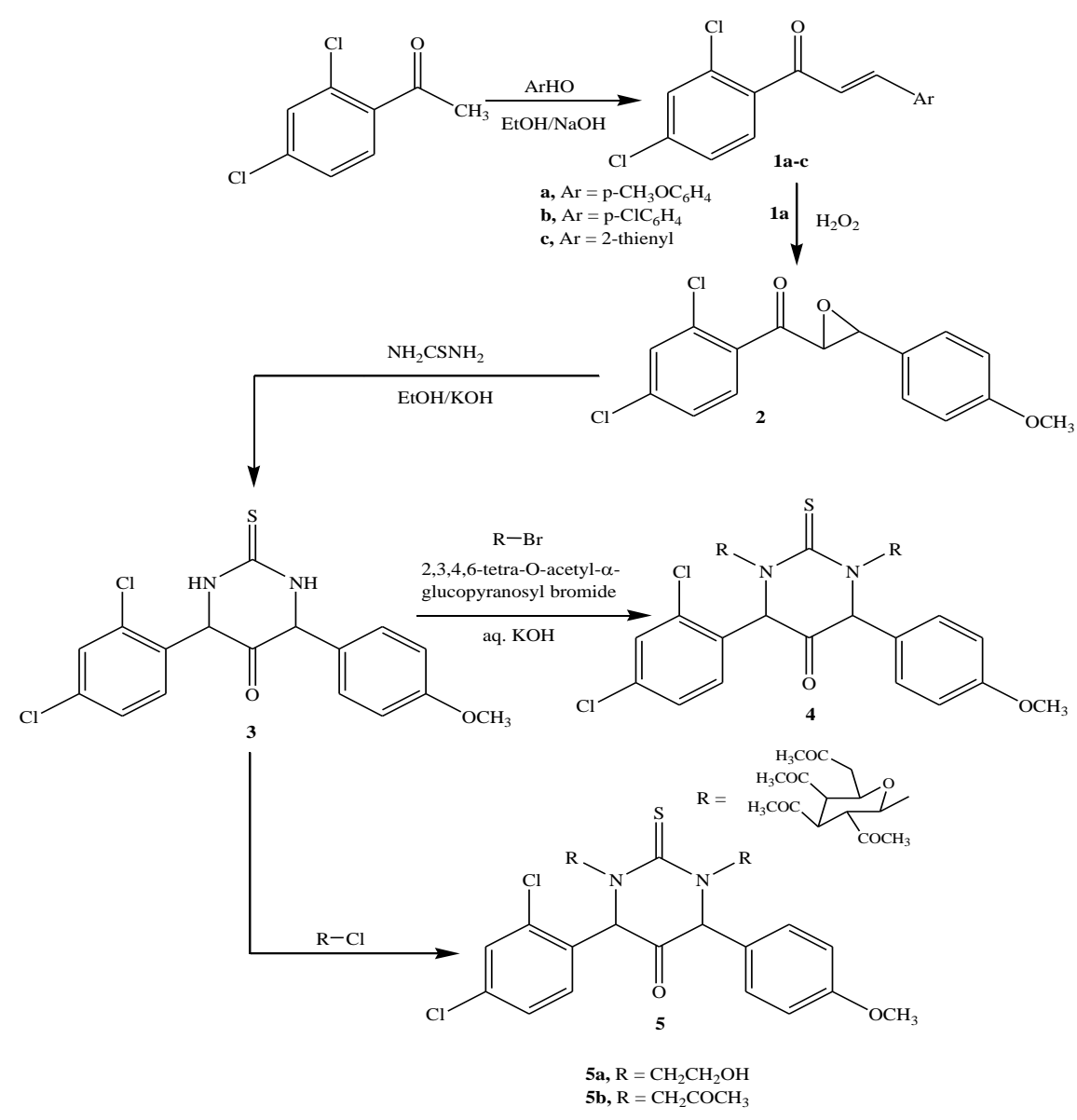

Scheme 1

Chalcone $1 \mathrm{~b}$ was treated with acetyl acetone in the presence of alcoholic sodium hydroxide or hydrazine hydrate in refluxing ethanol to give cyclic Michael addition product 6 and 3-(2,4-dichlorophenyl)-5-(p-chloro-phenyl)-4,4dihydropyrazoline 7 , respectively. While, compounds 1a-c were condensed with urea or thiourea in refluxing ethanolic potassium hydroxide to afford the corresponding pyrimidine derivatives $8 \mathrm{a}-\mathrm{c}$ and $9 \mathrm{a}-\mathrm{c}$, respectively. Also, the reaction of $1 \mathrm{a}, \mathrm{b}$ with thiosemicabazide gave the aminopyrimidinethiones $10 \mathrm{a}, \mathrm{b}$, respectively. The latter compound $10 \mathrm{~b}$ was reacted with p-chlorobenzaldehyde or 2-chloroethanol to give the corresponding Schiff base 11 and 4-(4-chlorophenyl)-6-(2,4-dichloro-phenyl)-1-(2-hydroxy-ethyl)-3-(2-hydroxyethylamino)- 
3,4-dihydro-1H-pyrimidine-2-thione (12), respectively (Scheme 2).

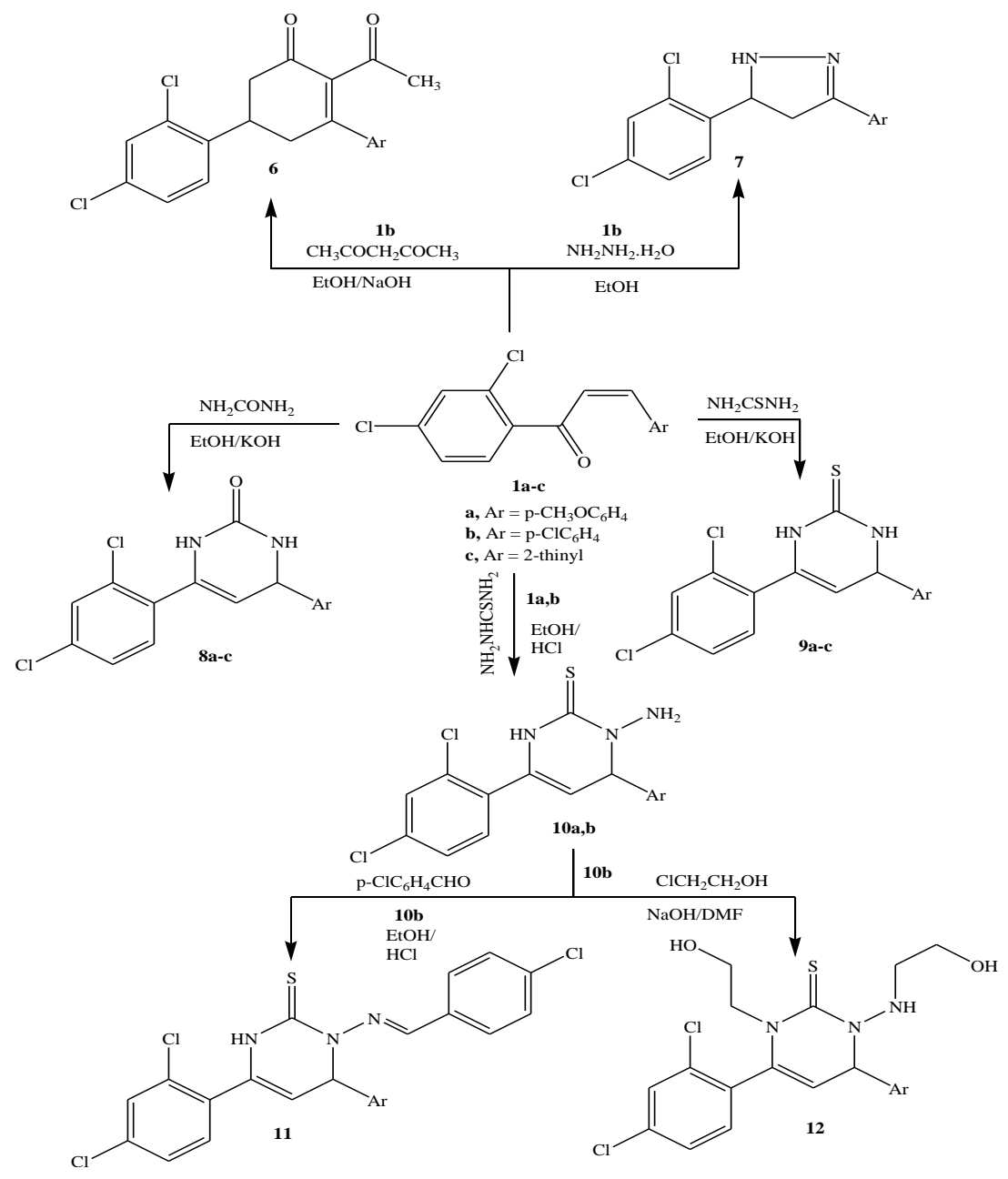

Scheme 2

Finally, compound 9a was reacted with methyl iodide to afford S-methyl derivative 13 and when compound 9a was reacted with and ethylchloroacetate in refluxing alcoholic potassium hydroxide to afford compound 14. Also, compound 9a was treated with bromoacetic acid and p-methoxybenzaldehyde in the presence of anhydrous sodium acetate to afford the corresponding thiazolopyrimidine derivative 15 (Scheme 3).

Egypt. J. Chem. 55, No.4 (2012) 


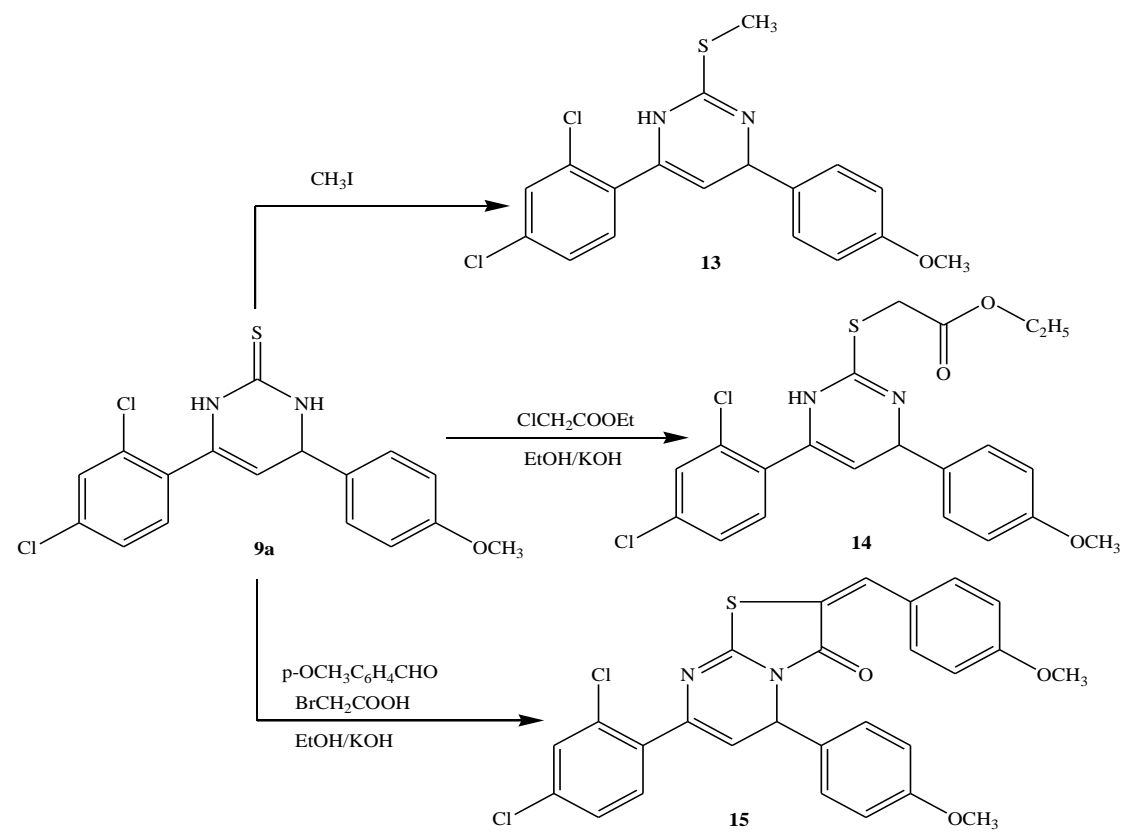

Scheme 3

Biological evaluation

Antioxidant activity

The antioxidant activity of chalcone 1a, oxiranyl methanone 2, thioxoprymidinone 5a, acetylcyclohexnone 6 , pyrazole 7 , pyrimidine-2-thione 10a which were prepared and selected for screening against the artificial radical DPPH is shown in Fig. $1 \& 2$. Pyrimidinethione 10a and pyrazole 7 have exhibited a high scavenging activity compared to the other compounds.

From the chemical point of view, the enhanced activity evaluated for the amino-prymidinethione derivative 10 a may be attributed to the delocalization of the developing radical on the nitrogen and sulfur atom with the double bond in the ring leading to further stabilization.

\section{Molecular docking study}

Docking using autodock vina

The crystal structure of Tyrosine Kinase was downloaded from protein data bank (http://www.pdb.org/pdb/ ) pdb code $=1 \mathrm{t} 46$. The site in which the reported inhibitor was determined by using pdbsum (http://www.ebi.ac.uk/thorntonsrv/databases/cgi-bin/pdbsum/GetPage.pl) as exhibited in Fig. 3.

Egypt. J. Chem. 55, No.4 (2012) 


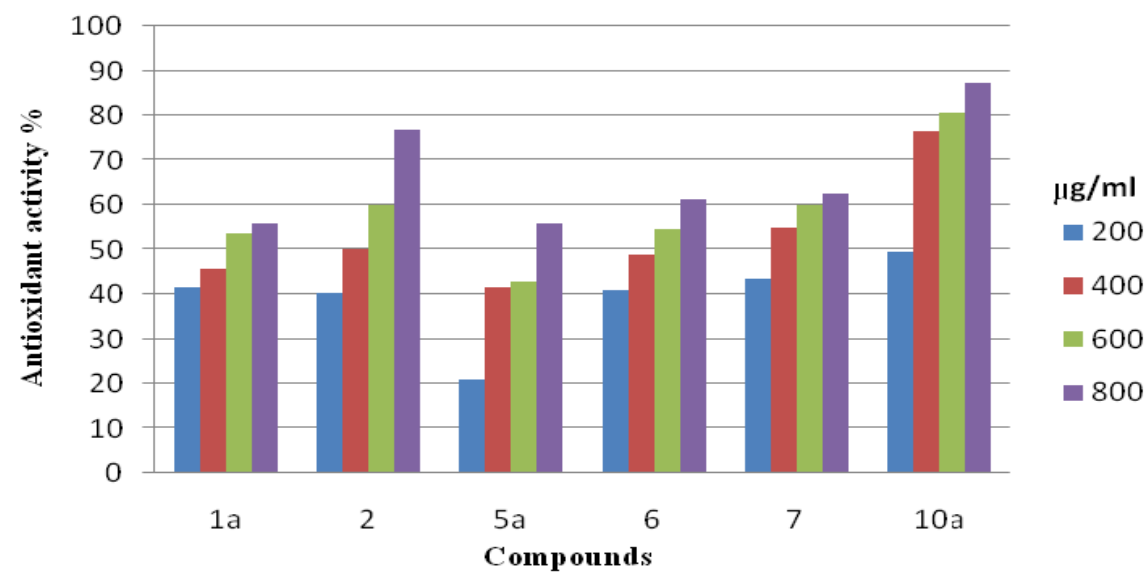

Fig. 1. Antioxidant activity $\%$ of different synthetic compounds against DPPH radical assay.

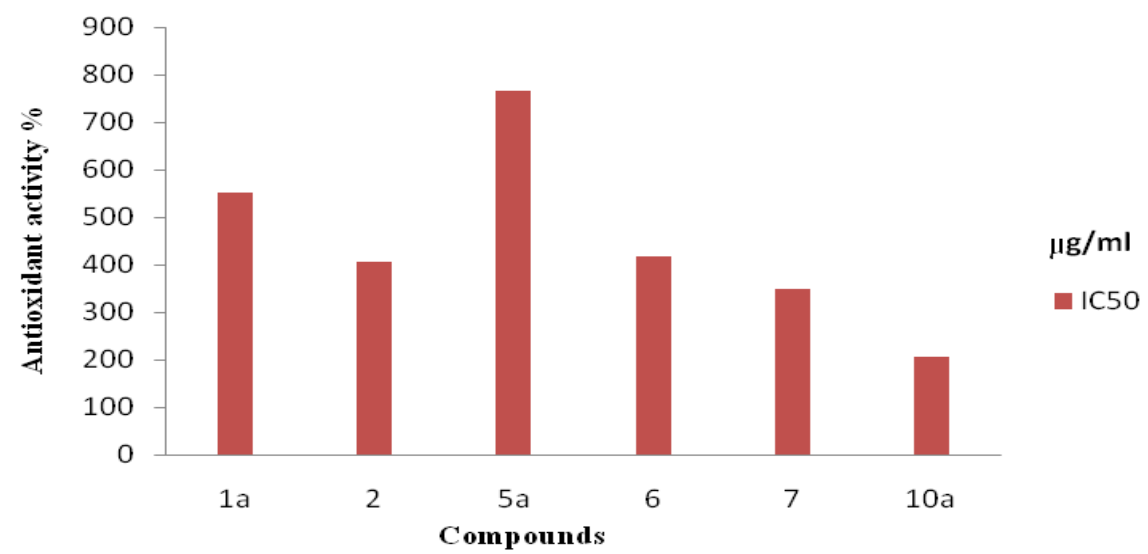

Fig. 2. Antioxidant activity $\%$ against $\mathrm{IC}_{50}(\mu \mathrm{g} / \mathrm{ml})$ for different synthetic chemical compounds.

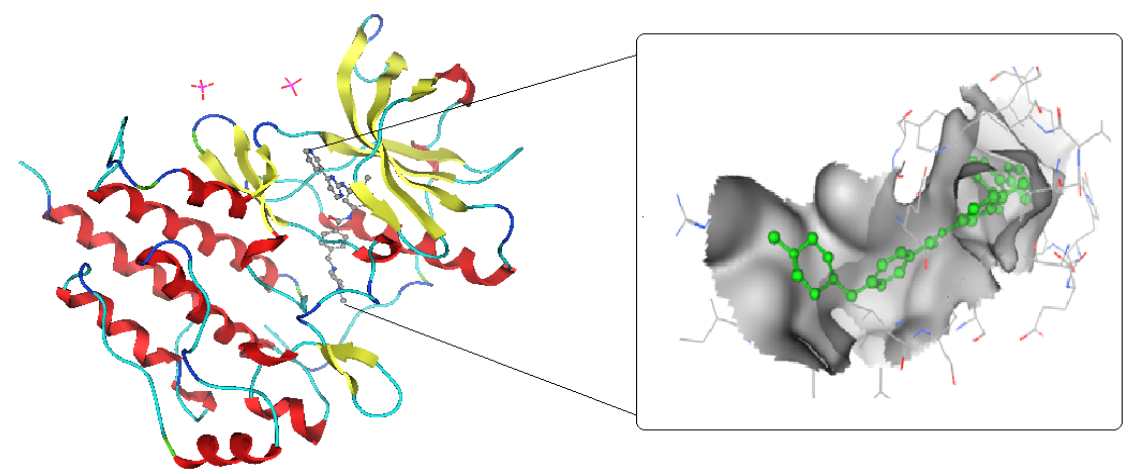

Egypt. J. Chem. 55, No.4 (2012) 
Fig. 3. Visualization the active site at which the inhibitor complexed.

The important residues that form interactions with that inhibitor were determined by pdbsum as well. It was found that Cys 673, Thr 670, Glu 640 and Asp 810 were forming some interactions with different moieties of the inhibitor as shown in Fig. 4.

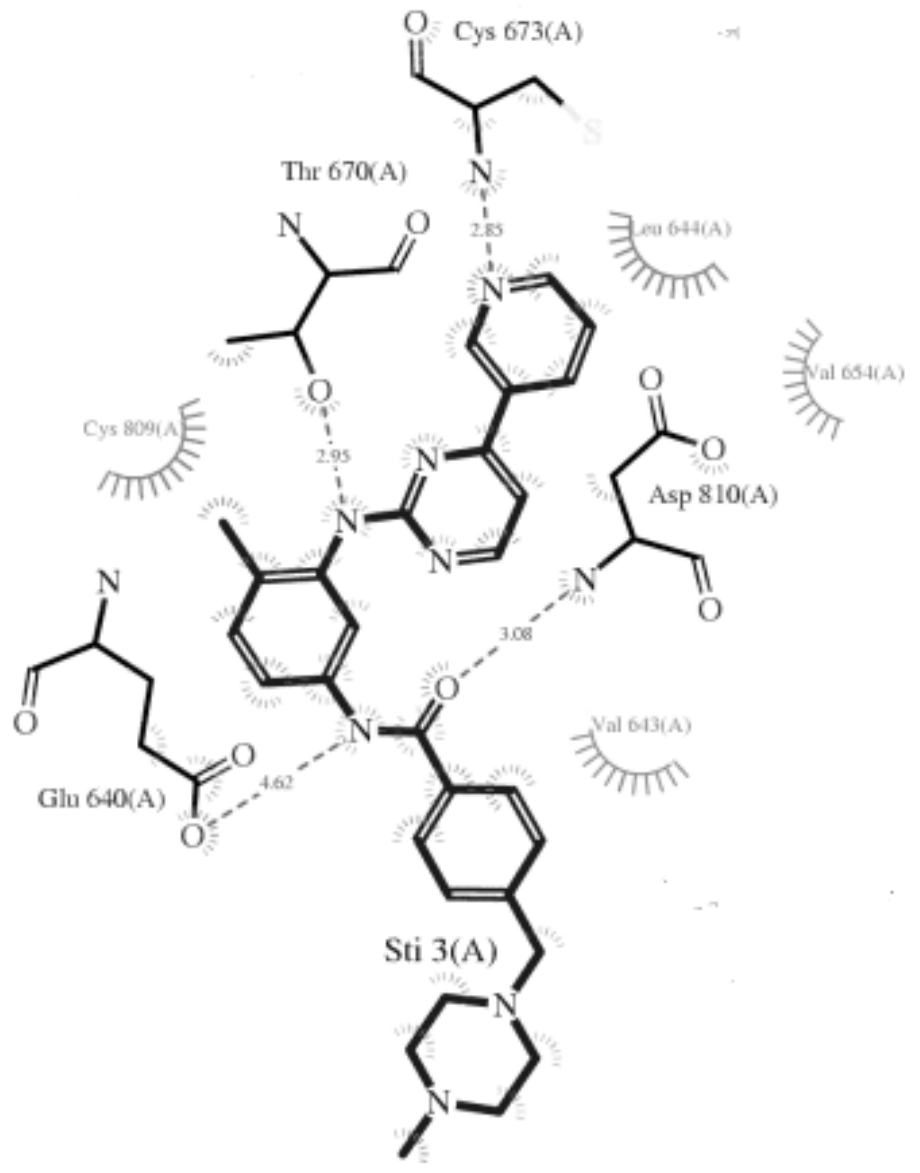

Fig. 4. An image taken from Pdbsum showing the most visible interactions that were formed with the complexed ligand.

The affinity of any small molecule can be considered to be a unique tool in the field of drug design. As there is a relationship between the affinity of organic molecules and the free binding energy and this can contribute in prediction and interpretation of the activity of organic compounds toward a specific target protein. Here we used Autodock Vina program for docking and listed the affinities and the most visible interactions for all of compounds in Table 1.

Egypt. J. Chem. 55, No.4 (2012) 
TABLE 1. The calculated affinities and most visible interactions for the synthesized compounds.

\begin{tabular}{|c|c|c|c|}
\hline Compound & $\begin{array}{l}\text { Affinity } \\
\text { kcal/mol }\end{array}$ & $\begin{array}{l}\text { Most visible } \\
\text { interaction }\end{array}$ & $\begin{array}{l}\text { Important moiety } \\
\text { of the compound }\end{array}$ \\
\hline $1 \mathrm{a}$ & -10.76 & Cys 673 & $\mathrm{C}=\mathrm{O}$ \\
\hline $1 b$ & -9.55 & Asp 810 & $\mathrm{C}=\mathrm{O}, \mathrm{C}=\mathrm{C}$ \\
\hline 2 & -10.6 & $\begin{array}{c}\text { Thr 670, Cys } 673 \\
\text { Asp } 810\end{array}$ & Oxygen atom \\
\hline 3 & -10.77 & Asp 810 & $\mathrm{C}=\mathrm{O}, \mathrm{C}=\mathrm{S}$ \\
\hline $5 \mathrm{a}$ & -7.5 & $\ldots \ldots \ldots$ & $\mathrm{C}=\mathrm{O}$ \\
\hline $5 b$ & -8.7 & Asp 810 & $\mathrm{CH} 3-\mathrm{CO}$ \\
\hline 6 & -11.6 & Cys 673 (-NH) & Oxygen atom \\
\hline 7 & -12.19 & $\begin{array}{l}\text { Glu } 640(\mathrm{C}=\mathrm{O}) \\
\text { Lys } 623(-\mathrm{NH})\end{array}$ & pyrazole ring \\
\hline $8 \mathrm{a}$ & -9.00 & As0 $810(\mathrm{C}=\mathrm{O})$ & $\mathrm{C}=\mathrm{N}$ \\
\hline $8 \mathrm{~b}$ & -9.3 & Thr 670 & $\mathrm{C}=\mathrm{O}$ \\
\hline $8 \mathrm{c}$ & -11.98 & & $\mathrm{HN}-\mathrm{CO}-\mathrm{NH}$ \\
\hline $9 b$ & -10.93 & As0 $810(\mathrm{C}=\mathrm{O})$ & $\mathrm{C}=\mathrm{O}$ \\
\hline $9 \mathrm{c}$ & -12.05 & & $\mathrm{C}=\mathrm{S}$ \\
\hline $10 \mathrm{a}$ & -12.09 & $\begin{array}{c}\text { Glu } 640(\mathrm{C}=\mathrm{O}) \\
\text { Lys } 623 \\
\end{array}$ & $\mathrm{C}=\mathrm{S}$ \\
\hline $10 \mathrm{~b}$ & -10.07 & Glu $640(\mathrm{C}=\mathrm{O})$ & $\mathrm{C}=\mathrm{S}$ \\
\hline 11 & -8.10 & Asp $810(\mathrm{C}=\mathrm{O})$ & $\mathrm{C}=\mathrm{S}, \mathrm{NH}$ \\
\hline 12 & -8.63 & Lys 623 & Oxygen atom \\
\hline 14 & -8.13 & Asp $810(\mathrm{C}=\mathrm{O})$ & $\mathrm{C}=\mathrm{O}$ \\
\hline 15 & -10.73 & Cys 673 (-NH) & $\mathrm{C}=\mathrm{O}$ \\
\hline
\end{tabular}

Inspection of the Table 1 revealed that, that compounds 1a, 2, 3, 6, 7, 9b, 10a, $10 \mathrm{~b}$ and 15 have the best affinity values ranging from -10.07 to $-12.19 \mathrm{kcal} / \mathrm{mol}$.

The tested compounds have showed a good fitting inside the active site of TK. The best affinity of $-12.19 \mathrm{kcal} / \mathrm{mol}$. was recorded to 7 . The visualized docking site of 7 compared to the reference ligand was found complexed with the enzyme through the interactions with the residues such as Glu and Asp as found in pdbsum as shown in Fig. 5. 


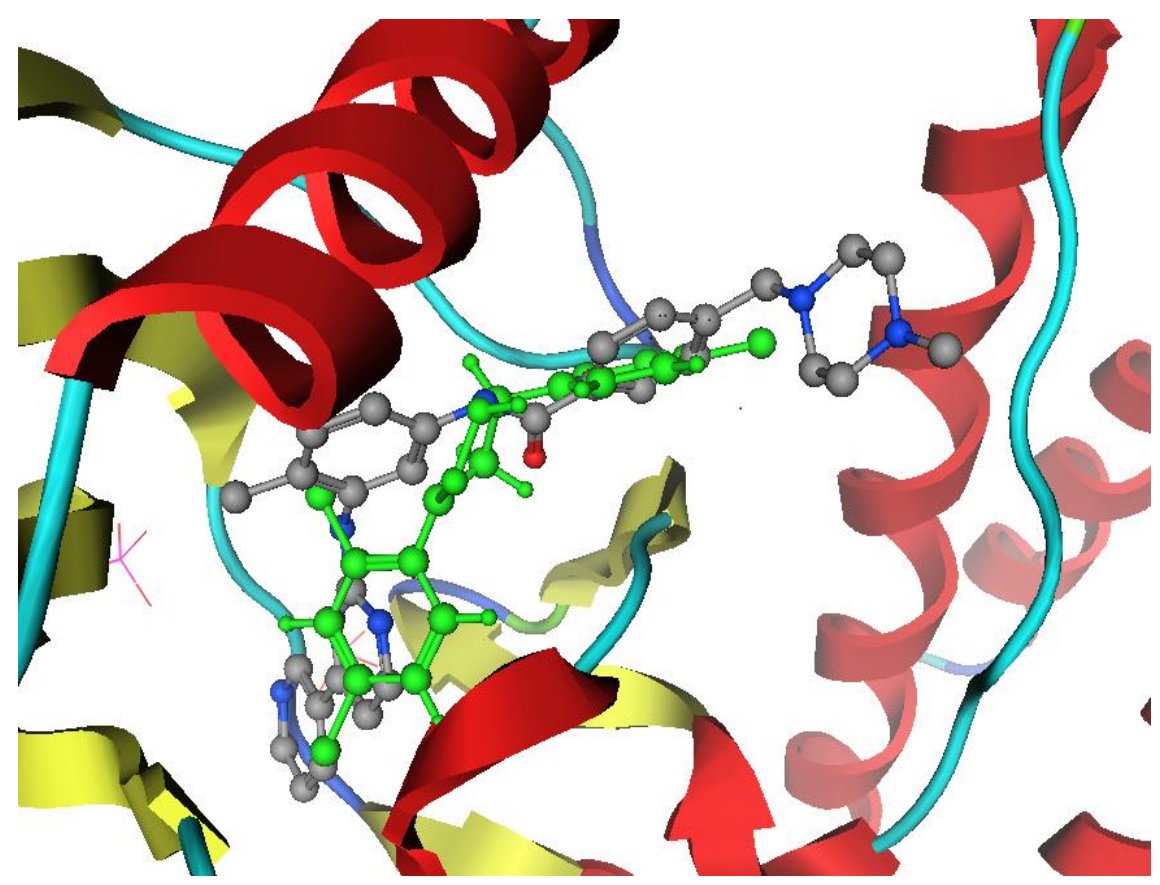

Fig. 5. Compound 7 (represented as element) fits in the same site and that of the reference ligand (colored green).

\section{Experimental}

Melting points were measured using Electrothermal IA 9100 apparatus, (Shimadzu, Tokyo, Japan). IR spectra were recorded as potassium bromide pellets on a Perkin-Elmer 1650 spectrophotometer (Perkin-Elmer, Norwalk, CT, USA). ${ }^{1} \mathrm{H}$ NMR was determined on a Jeol-Ex-300 expressed as part per million; ppm ( $\delta$ values) against TMS as internal reference. Mass spectra were recorded on VG 2AM-3F mass spectrometer (Thermo electron Corporation, USA). Microanalyses were operated using Mario El Mentar apparatus, Organic Microanalysis Unit. Follow up of the reactions and checking the purity of the compounds was made by TLC on silica gel-precoted aluminum sheets (Type 60 F254, Merck, and Darmstadt, Germany).

3-(Substituted)-1-(2,4-dichlorophenyl)-propen-1-one (1a,c).

To a solution of 2,4-dichloroacetophenone $(1.88 \mathrm{~g}, 0.01 \mathrm{~mol})$ and aldehydes, namely, p-methoxybenzaldehyde, p-chlorobenzaldehyde or thiophene-2carbaldehyde $(0.01 \mathrm{~mol})$ in ethanol $30 \mathrm{ml}, \mathrm{NaOH}(10 \%, 15 \mathrm{ml})$ was added drop wise within $15 \mathrm{~min}$. The reaction mixture was stirred for $3 \mathrm{hr}$ and left overnight at room temperature, then poured onto ice. The obtained precipitate was filtered off, dried and crystallized from ethanol to give $(1 \mathrm{a}, \mathrm{c})$, respectively. 
1-(2,4-Dichlorophenyl)-3-(4-methoxyphenyl)propen-1-one (1a)

Yield 85\%; m.p.122-124 ${ }^{\circ} \mathrm{C}$; IR (KBr, v, $\left.\mathrm{cm}^{-1}\right)$ : $1677(\mathrm{C}=\mathrm{O}) ;{ }^{1} \mathrm{H}$ NMR $\left(\right.$ DMSO-d $\left._{6}, \delta \mathrm{ppm}\right): 3.75\left(\mathrm{~s}, 3 \mathrm{H}, \mathrm{OCH}_{3}\right), 7.18(\mathrm{~d}, J=2.4 \mathrm{~Hz}, 1 \mathrm{H}$, olefinic $\mathrm{H}), 7.40$ (d, J=2.6 Hz, 1H, olefinic H), 7.59-8.18 (m, 7H, Ar-H); MS m/z (\%): $307\left(\mathrm{M}^{+}\right.$, 62), $309\left(\mathrm{M}^{+}+2,43\right)$, Analysis for $\mathrm{C}_{16} \mathrm{H}_{12} \mathrm{Cl}_{2} \mathrm{O}_{2}(307.17)$ : required $\mathrm{C}, 62.56 ; \mathrm{H}$, 3.94; $\mathrm{Cl}, 23.08$; found $\mathrm{C}, 62.52 ; \mathrm{H}, 3.88 ; \mathrm{Cl}, 23.00$.

\section{1-(2,4-Dichlorophenyl)-3-(thiophen-2-yl)-propen-1-one (1c)}

Yield 62\%; m.p. 94-96 ${ }^{\circ} \mathrm{C}$; IR $\left(\mathrm{KBr}, v, \mathrm{~cm}^{-1}\right)$ : $1672(\mathrm{C}=\mathrm{O})$; ${ }^{1} \mathrm{H}$ NMR (DMSO-d $\left.\mathrm{D}_{6}, \delta \mathrm{ppm}\right): 7.14(\mathrm{~d}, J=2.7 \mathrm{~Hz}, 1 \mathrm{H}$, olefinic $\mathrm{H}), 7.30(\mathrm{~d}, J=2.65 \mathrm{~Hz}, 1 \mathrm{H}$, olefinic H), 7.04-8.11 (m, 6H, Ar-H); MS: m/z (\%): $283\left(\mathrm{M}^{+}, 100\right), 285\left(\mathrm{M}^{+}+2\right.$, 71). Analysis for $\mathrm{C}_{13} \mathrm{H}_{8} \mathrm{Cl}_{2} \mathrm{OS}$ (283.17): required $\mathrm{C}, 55.14 ; \mathrm{H}, 2.85 ; \mathrm{Cl}, 25.04 ; \mathrm{S}$, 11.32 found $\mathrm{C}, 55.10 ; \mathrm{H}, 2.79 ; \mathrm{Cl}, 24.94 ; \mathrm{S}, 11.21$.

\section{(2,4-Dichlorophenyl)-3-(4-methoxyphenyl)oxiran-2-yl)methanone (2)}

Hydrogen peroxide $(5 \mathrm{ml}, 30 \%)$ was added portion wise to a mixture of 3-(4methoxyphenyl)-1-(3,4-dichloro-phenyl)-propenone $1 \mathrm{a}(0.01 \mathrm{~mol})$ in acetone (50 $\mathrm{ml})$, and methanol $(15 \mathrm{ml})$ containing $\mathrm{NaOH}(1 \mathrm{~g})$ at $20-25^{\circ} \mathrm{C}$ with stirring. The reaction mixture was left over night, the precipitated solid was filtered off, washed with cold water and crystallized from ethanol to give compound 2. Yield $75 \%$; m.p 100-102 ${ }^{\circ} \mathrm{C}$, (ethanol), IR $\left(\mathrm{KBr}, v, \mathrm{~cm}^{-1}\right)$ : $1700(\mathrm{C}=\mathrm{O}) ;{ }^{1} \mathrm{H}$ NMR spectrum (DMSO-d $6, \delta$ ppm): $3.75\left(\mathrm{~s}, 3 \mathrm{H}, \mathrm{OCH}_{3}\right), 4.10,(\mathrm{~d}, 1 \mathrm{H}, J=2.4 \mathrm{~Hz}$, epoxy-H), 4.48, (d, 1H, J=2.38 Hz, epoxy-H), 7.00-7.82 (m, $7 \mathrm{H}, \mathrm{Ar}-\mathrm{H})$; MS: $\mathrm{m} / \mathrm{z}(\%): 323\left(\mathrm{M}^{+}, 100\right), 325\left(\mathrm{M}^{+}+2,72\right)$. Analysis for $\mathrm{C}_{16} \mathrm{H}_{12} \mathrm{Cl}_{2} \mathrm{O}_{3}$ (323.17): required $\mathrm{C}, 59.46 ; \mathrm{H}, 3.74 ; \mathrm{Cl}, 21.94$; found $\mathrm{C}, 59.39 ; \mathrm{H}, 3.68 ; \mathrm{Cl}, 21.90$.

4-(2,4-Dichloro -phenyl)-6- (4-Methoxy-phenyl) -2-thioxotetrahydro-pyrimidin $5(6 \mathrm{H})$-one (3)

A mixture of compound $2(0.01 \mathrm{~mol})$ and thiourea $(0.02 \mathrm{~mol})$ in ethanolic potassium hydroxide ( $2 \mathrm{~g}$ in $100 \mathrm{ml}$ ethanol) was refluxed for $4 \mathrm{hr}$. The solvent was evaporated and the formed solid was washed several times with acidified cold water, filtered off and recrystallized from methanol to give compound 3 . Yield 77\%, m.p.174-176 ${ }^{\circ} \mathrm{C}$, IR $\left(\mathrm{KBr}, v, \mathrm{~cm}^{-1}\right)$ : 3135-3216 (2NH), $1250(\mathrm{C}=\mathrm{S})$, $1719(\mathrm{C}=\mathrm{O})$; ${ }^{1} \mathrm{H}$ NMR (DMSO-d $\left.6, \delta \mathrm{ppm}\right): 3.6\left(\mathrm{~s}, 3 \mathrm{H}, \mathrm{OCH}_{3}\right), 4.01(\mathrm{~s}, 1 \mathrm{H}$, pyrimidine-H), 4.2 (s, 1H, pyrimidine-H), 6.94-7.92 (m, $7 \mathrm{H}, \mathrm{Ar}-\mathrm{H}), 9.8(\mathrm{~s}, 1 \mathrm{H}$, $\mathrm{NH}, \mathrm{D}_{2} \mathrm{O}$ exchangeable), 10.9 (s, $1 \mathrm{H}, \mathrm{NH}, \mathrm{D}_{2} \mathrm{O}$ exchangeable); ${ }^{13} \mathrm{CNMR}: 54.8$, $\mathrm{OCH}_{3}, 63.2,71.8 \mathrm{sp}^{3}$ carbon, 126.2-147.5, aromatic carbons, $176.8(\mathrm{C}=\mathrm{O}), 200.9$ $(\mathrm{C}=\mathrm{S})$; $\mathrm{MS}, \quad m / z \quad(\%)$ : $381 \quad\left(\mathrm{M}^{+}, 62\right), 383 \quad\left(\mathrm{M}^{+}+2,41\right)$. Analysis for $\mathrm{C}_{17} \mathrm{H}_{14} \mathrm{Cl}_{2} \mathrm{~N}_{2} \mathrm{O}_{2} \mathrm{~S}$ (381.28): required C, 53.55; H, 3.70; Cl, 18.60; N, 7.35; S, 8.41; found $\mathrm{C}, 53.25 ; \mathrm{H}, 3.51 ; \mathrm{Cl}, 18.39 ; \mathrm{N}, 7.14 ; \mathrm{S}, 8.39$.

4-(4-Methoxy-phenyl)-6-(2,4-dichloro-phenyl)-1,3-di-(2,3,4,6-tetra-O-acetyl- $\beta$ D-glucopyranosyl) -2-thioxo-tetrahydropyrimidin-5-one (4)

To a solution of compound $3(0.01 \mathrm{~mol})$ in aqueous $\mathrm{KOH}(0.56 \mathrm{~g}, 0.01 \mathrm{~mol})$ in distilled water $(5 \mathrm{ml})$, a solution of 2,3,4,6-tetra- $O$-acetyl- $\alpha$-glucopyranosyl bromide $(0.02 \mathrm{~mol})$ in $5 \mathrm{ml}$ acetone was added. The reaction mixture was stirred for $16 \mathrm{hr}$ at room temperature under TLC control, the solvent was evaporated Egypt. J. Chem. 55, No.4 (2012) 
under reduced pressure at $40^{\circ} \mathrm{C}$. The obtained residue was solidified and filtered off, washed with distilled water and crystallized from diethyl ether as a yellow powder 4. Yield $77 \%$, m.p. $133-135^{\circ} \mathrm{C}$, IR $\left(\mathrm{KBr}, v, \mathrm{~cm}^{-1}\right): 1713(\mathrm{C}=\mathrm{O})$ and 1750 $(\mathrm{C}=\mathrm{O}) .{ }^{1} \mathrm{H}$ NMR (DMSO-d 6 , $\delta$ ppm):1.89-2.09 (8s, 24H, 8CH $\mathrm{CO}_{3} \mathrm{CO}, 3.71(\mathrm{~s}, 3 \mathrm{H}$, $\left.\mathrm{OCH}_{3}\right), 4.00-4.11\left(\mathrm{~m}, 6 \mathrm{H}, 5^{\prime}-\mathrm{H}, 5^{\prime \prime}-\mathrm{H}, 66^{\prime}-\mathrm{H}_{2}, 6^{\prime \prime}-\mathrm{H}_{2}\right), 4.24$ (s, 1H, pyrimidine-H), 4.66 (s, 1H, pyrimidine-H), 4.95-5.30 (m, 7H, 2'-H, 2"-H, 3'-H, 3"-H, 4'-H, 4"-H, $1 '-\mathrm{H})$, and $5.70(\mathrm{~d}, 1 \mathrm{H}, J=9.8 \mathrm{~Hz}, 1 "-\mathrm{H})$.

6-(2,4-Dichlorophenyl) -tetrahydro-1,3- bis(substituted) -4-(4-methoxyphenyl)2-thioxopyrimidin-5(6H)-one $(5 a, b)$

To a solution of compound $3(0.01 \mathrm{~mol})$ in dry DMF $(20 \mathrm{ml})$, sodium hydroxide $(0.24 \mathrm{~g}, 0.01 \mathrm{~mol})$ was added. The reaction mixture was stirred at $70^{\circ} \mathrm{C}$ for $3 \mathrm{hr}$, cooled and then, chlorethanol or chloroacetone $(0.01 \mathrm{~mol})$ was added with stirring at room temperature for $5 \mathrm{hr}$. The reaction mixture was evaporated under reduced pressure, the formed residue was washed with distilled water, filtered off, dried and crystallized from proper solvent to give compounds (5a, b), respectively.

6-(2,4-Dichlorophenyl)- tetrahydro-1,3-bis (2-hydroxyethyl) -4-(4-methoxyphenyl) -2- thioxopyrimidin-5(6H)-one (5a)

Yield 65\%, m.p.116-118 ${ }^{\circ} \mathrm{C}$, IR (KBr, v, $\left.\mathrm{cm}^{-1}\right)$ : $1222(\mathrm{C}=\mathrm{S}), 1709(\mathrm{C}=\mathrm{O}), 3418$ $(\mathrm{OH}),{ }^{1} \mathrm{H}$ NMR (DMSO-d 6 , $\left.8 \mathrm{ppm}\right): 3.10\left(\mathrm{t}, 4 \mathrm{H}, 2 \mathrm{CH}_{2}\right), 3.55\left(\mathrm{t}, 4 \mathrm{H}, 2 \mathrm{CH}_{2}\right), 3.75(\mathrm{~s}$, $\left.3 \mathrm{H}, \mathrm{OCH}_{3}\right), 4.2$ (br, $2 \mathrm{H}, 2 \mathrm{OH}, \mathrm{D}_{2} \mathrm{O}$ exchangeable), $4.5(\mathrm{~s}, 1 \mathrm{H}$, pyrimidine), $4.7(\mathrm{~s}, 1 \mathrm{H}$, pyrimidine), 6.9-7.98 (m, $7 \mathrm{H}, \mathrm{Ar}-\mathrm{H})$; MS: m/z (\%): $469\left(\mathrm{M}^{+}, 100\right), 471\left(\mathrm{M}^{+}+2,73\right)$. Analysis for $\mathrm{C}_{21} \mathrm{H}_{22} \mathrm{Cl}_{2} \mathrm{~N}_{2} \mathrm{O}_{4} \mathrm{~S}(469.38)$ : required $\mathrm{C}, 53.74 ; \mathrm{H}, 4.72 ; \mathrm{Cl}, 15.11 ; \mathrm{N}$, 5.97; S, 6.83; found C, 53.71; H, 4.66; Cl, 14.98; N, 5.87; S, 6.80 .

6-(2,4-Dichlorophenyl)-tetrahydro-4-(4-methoxyphenyl)-1,3-bis(2-oxopropyl) -2-thioxo-pyrimidin-5(6H)-one (5b)

Yield 68\%, m.p. $125-127{ }^{\circ} \mathrm{C}$, IR (KBr, v, $\left.\mathrm{cm}^{-1}\right)$ : $1221(\mathrm{C}=\mathrm{S}), 1696,1698$ $1731(3 \mathrm{C}=\mathrm{O}),{ }^{1} \mathrm{H}$ NMR (DMSO-d 6 , $\left.\delta \mathrm{ppm}\right): 1.80\left(\mathrm{~s}, 6 \mathrm{H}, 2 \mathrm{CH}_{3}\right), 2.95(\mathrm{~s}, 4 \mathrm{H}$, $\left.2 \mathrm{CH}_{2}\right), 3.72\left(\mathrm{~s}, 3 \mathrm{H}, \mathrm{OCH}_{3}\right), 4.40(\mathrm{~s}, 1 \mathrm{H}$, pyrimidine $), 4.74(\mathrm{~s}, 1 \mathrm{H}$, pyrimidine $)$, 6.9-8.1 (m, 7H, Ar-H); MS: m/z (\%): $493(\mathrm{M}+, 100), 495\left(\mathrm{M}^{+}+2,70\right)$. Analysis for $\mathrm{C}_{23} \mathrm{H}_{22} \mathrm{Cl}_{2} \mathrm{~N}_{2} \mathrm{O}_{4} \mathrm{~S}$ (493.40): required $\mathrm{C}, 55.99 ; \mathrm{H}, 4.49 ; \mathrm{Cl}, 14.37 ; \mathrm{N}, 5.68 ; \mathrm{S}$, 6.50; found C, 55.86; H, 4.39; Cl, 14.23; N, 5.61; S, 6.44.

2-Acetyl-3-(4-chloro-phenyl)-5-(2,4-dichloro-phenyl)-cyclohex-2-enone (6)

A mixture of compound $1 \mathrm{~b}(3.11 \mathrm{~g}, 0.01 \mathrm{~mol})$, acetyl acetone $(1.0 \mathrm{~g}, 0.01$ mol) in ethanol and $\mathrm{NaOH}(50 \%, 15 \mathrm{ml})$ was refluxed for $6 \mathrm{hr}$. The reaction mixture was poured onto ice water and acidified with conc. $\mathrm{HCl}$ to give a solid which was crystallized from dioxane to give compound 6 . Yield 70\%, m.p. 145$146{ }^{\circ} \mathrm{C}$; IR (KBr, $\left.v, \mathrm{~cm}^{-1}\right)$ : 1695 and $1702(2 \mathrm{C}=\mathrm{O}) ;{ }^{1} \mathrm{H}$ NMR (DMSO-d $6, \delta$ ppm): $1.3\left(\mathrm{~d}, 2 \mathrm{H}, J=3.05 \mathrm{~Hz}, \mathrm{CH}_{2}\right), 1.8(\mathrm{~m}, 1 \mathrm{H}, \mathrm{CH}), 2.6\left(\mathrm{~d}, 2 \mathrm{H}, J=2.11 \mathrm{~Hz}, \mathrm{CH}_{2}\right), 2.78$ (s, 3H, $\left.\mathrm{CH}_{3}\right), 6.9-7.9(\mathrm{~m}, 7 \mathrm{H}, \mathrm{Ar}-\mathrm{H})$; $\mathrm{MS}: \mathrm{m} / \mathrm{z}(\%): 394\left(\mathrm{M}^{+}, 30\right), 396\left(\mathrm{M}^{+}+2,28\right)$, Analysis for $\mathrm{C}_{20} \mathrm{H}_{15} \mathrm{Cl}_{3} \mathrm{O}_{2}$ (393.69): required $\mathrm{C}, 61.02 ; \mathrm{H}, 3.84 ; \mathrm{Cl}, 27.02$; found C, 61.00; H, 3.72; Cl, 26.87. 
3-(4-Chlorophenyl)-5-(2,4-dichlorophenyl)-4,5-dihydro-1H-pyrazole (7)

A solution of chalcone $1 \mathrm{~b}(3.11 \mathrm{~g}, 0.01 \mathrm{~mol})$ and hydrazine hydrate $(0.32 \mathrm{~g}$, $0.01 \mathrm{~mol}$ ) in $30 \mathrm{ml}$ absolute ethanol was refluxed for $5 \mathrm{hr}$ and then concentrated under reduced pressure. The obtained precipitate was filtered off, dried and crystallized from methanol to give the pyrazoline (7). Yield 70\%; m.p. 130$131{ }^{\circ} \mathrm{C}$; IR $\left(\mathrm{KBr}, v, \mathrm{~cm}^{-1}\right): 3214(\mathrm{NH}) ;{ }^{1} \mathrm{H}$ NMR (DMSO-d $\left.{ }_{6}, \delta \mathrm{ppm}\right): 6.7(\mathrm{~d}, 1 \mathrm{H}$, $\left.J=2.4 \mathrm{~Hz}, \mathrm{CH}_{2}\right), 6.9$ (d, 1H, J=2.2 Hz, CH-Ar), 7.2-7.7 (m, 7H, Ar-H), $9.8(\mathrm{~s}, 1 \mathrm{H}$, $\mathrm{NH}, \mathrm{D}_{2} \mathrm{O}$ exchangeable ); $\mathrm{Ms}, \mathrm{m} / \mathrm{z}(\%): 325\left(\mathrm{M}^{+}, 33\right), 327\left(\mathrm{M}^{+}+2,32\right)$. Analysis for $\mathrm{C}_{15} \mathrm{H}_{11} \mathrm{Cl}_{3} \mathrm{~N}_{2}$ (325.62): required $\mathrm{C}, 55.32 ; \mathrm{H}, 3.40 ; \mathrm{Cl}, 32.66 ; \mathrm{N}, 8.6$; found $\mathrm{C}$, $55.11 ; \mathrm{H}, 3.54 ; \mathrm{Cl}, 32.75 ; \mathrm{N}, 8.48$.

6-(2,4-Dichlorophenyl)-3,4-dihydro-4-( Substituted)pyrimidin-2(1H)-one (8a-c)

A solution of chalcone $1 \mathrm{a}-\mathrm{c}(0.01 \mathrm{~mol})$ and urea $(1 \mathrm{~g}, 0.015 \mathrm{~mol})$ was refluxed in $20 \mathrm{ml}$ acetic acid for $2 \mathrm{~h}$. The solid substance was filtered off and crystallized from acetic acid to give compounds (8a-c).

6-(2,4-Dichlorophenyl) -3,4-dihydro -4-(4-methoxyphenyl) pyrimidin-2(1H)one $(8 a)$

Yield 74\%; m.p. $200-202{ }^{\circ} \mathrm{C}$; IR $\left(\mathrm{KBr}, v, \mathrm{~cm}^{-1}\right)$ : (NH); Ms m/z (\%): 349 (M, 100), $351\left(\mathrm{M}^{+}+2\right.$, 69). Analysis for $\mathrm{C}_{17} \mathrm{H}_{14} \mathrm{Cl}_{2} \mathrm{~N}_{2} \mathrm{O}_{2}$ (349.21): required $\mathrm{C}, 58.47$; $\mathrm{H}, 4.04 ; \mathrm{Cl}, 20.30 ; \mathrm{N}, 8.02$; found C, 58.40; H, 3.98; Cl, 20.11; N, 7.92.

6-(2,4-Dichlorophenyl)-3,4-dihydro-4-(4-chlorophenyl)pyrimidin-2(1H)-one (8b) Yield 80\%; m.p. $177-179{ }^{\circ} \mathrm{C}$; IR (KBr, $\left.v, \mathrm{~cm}^{-1}\right)$ : (NH); Ms m/z (\%): $354\left(\mathrm{M}^{+}\right.$, 100), $356\left(\mathrm{M}^{+}+2,98\right)$. Analysis for $\mathrm{C}_{16} \mathrm{H}_{11} \mathrm{Cl}_{3} \mathrm{~N}_{2} \mathrm{O}$ (353.63): required $\mathrm{C}, 54.34$; $\mathrm{H}, 3.14 ; \mathrm{Cl}, 30.08 ; \mathrm{N}, 7.92$; found $\mathrm{C}, 54.27 ; \mathrm{H}, 3.01 ; \mathrm{Cl}, 29.93 ; \mathrm{N}, 7.87$.

6-(2,4-Dichlorophenyl)-3,4-dihydro-4-(thiophen-2-yl) pyrimidin-2(1H)-one (8c)

Yield 60\%; m.p.166-167 ${ }^{\circ} \mathrm{C}$; IR (KBr, v, cm $\left.{ }^{-1}\right)$ : $1702(\mathrm{C}=\mathrm{O}), 3220,3228$ $(2 \mathrm{NH}) ;{ }^{1} \mathrm{H}$ NMR (DMSO-d 6 , $\delta$ ppm): $4.6(\mathrm{~d}, 1 \mathrm{H}, J=3.2$, pyrimidine $), 5.2(\mathrm{~d}, 1 \mathrm{H}$, $J=3.22, \mathrm{~Hz}$, pyrimidine), 6.89-7.94 (m, 6H, Ar-H), $9.5\left(\mathrm{~s}, 1 \mathrm{H}, \mathrm{NH}, \mathrm{D}_{2} \mathrm{O}\right.$ exchangeable), $10.3\left(\mathrm{~s}, 1 \mathrm{H}, \mathrm{NH}, \mathrm{D}_{2} \mathrm{O}\right.$ exchangeable); $\mathrm{Ms} \mathrm{m} / \mathrm{z}(\%): 325\left(\mathrm{M}^{+}\right.$, 100), $327\left(\mathrm{M}^{+}+2,70\right)$. Analysis for $\mathrm{C}_{14} \mathrm{H}_{10} \mathrm{Cl}_{2} \mathrm{~N}_{2} \mathrm{OS}$ (325.21): required $\mathrm{C}, 51.70$; $\mathrm{H}, 3.10 ; \mathrm{Cl}, 21.80 ; \mathrm{N}, 8.61 ; \mathrm{S}, 9.86$; found $\mathrm{C}, 51.64 ; \mathrm{H}, 2.98 ; \mathrm{Cl}, 21.60 ; \mathrm{N}, 8.42$; S, 9.44 .

6-(2,4-Dichlorophenyl) -4-(substituted) -3,4-dihydropyrimidine -2(1H)-thione (9a-c)

A mixture of the chalcone $1 \mathrm{a}-\mathrm{c},(0.01 \mathrm{~mol})$, thiourea $(0.01 \mathrm{~mol})$ in alcoholic potassium hydroxide $(0.56 \mathrm{~g}, 0.01 \mathrm{~mol})$ in ethanol $(100 \mathrm{ml})$ was refluxed for $4 \mathrm{hr}$. The solvent was evaporated under reduced pressure and the obtained solid was suspended in distilled water several times, then filtered off, dried and crystallized from methanol to give the thiopyrimidine derivatives $(9 a-c)$.

6-(2,4-Dichlorophenyl) -4-(4-methoxyphenyl) -3,4-dihydropyrimidine -2(1H)thione (9a)

Yield 73\%; m.p. 187-188 ${ }^{\circ} \mathrm{C}$; IR (KBr, $\left.v, \mathrm{~cm}^{-1}\right)$ : 3154-3220 (NH); 1208 $(\mathrm{C}=\mathrm{S}) ;{ }^{1} \mathrm{HNMR}\left(\mathrm{DMSO}_{6}, \delta \mathrm{ppm}\right), 3.8\left(\mathrm{~s}, 1 \mathrm{H}, \mathrm{OCH}_{3}\right), 4.8(\mathrm{~d}, 1 \mathrm{H}, J=3.25 \mathrm{~Hz}$, Egypt. J. Chem. 55, No.4 (2012) 
pyrimidine), 5.7 (d, 1H, J=2.5 Hz, pyrimidine), 7.13-8.03 (m, 7H, Ar-H), 9.4 (s, $1 \mathrm{H}, \mathrm{NH}, \mathrm{D}_{2} \mathrm{O}$ exchangeable), $11.3\left(\mathrm{~s}, 1 \mathrm{H}, \mathrm{NH}, \mathrm{D}_{2} \mathrm{O}\right.$ exchangeable); $\mathrm{Ms} \mathrm{m} / \mathrm{z}(\%)$ : $365(\mathrm{M}, 100), 367\left(\mathrm{M}^{+}+2,72\right)$. Analysis for $\mathrm{C}_{17} \mathrm{H}_{14} \mathrm{Cl}_{2} \mathrm{~N}_{2} \mathrm{OS}$ (365.28): required C, 55.90; H, 3.86; Cl, 19.41; N, 7.67; S, 8.78; found C, 55.82; H, 3.75; Cl, 19.21; N, 7.44; S, 8.66.

6-(2,4-Dichlorophenyl) -4- (4-chlorophenyl) -3,4- dihydropyrimidine-2(1H)thione $(9 b)$

Yield 70\%; m.p. $137-138{ }^{\circ} \mathrm{C}$; IR (KBr, v, $\left.\mathrm{cm}^{-1}\right)$ : 3150-3200 (NH); 1200 $(\mathrm{C}=\mathrm{S}) ;{ }^{1} \mathrm{HNMR}$ (DMSO-d 6 , $\left.\delta \mathrm{ppm}\right), 4.7(\mathrm{~d}, 1 \mathrm{H}, \mathrm{J}=3.22 \mathrm{~Hz}$, pyrimidine), $5.2(\mathrm{~d}$, $1 \mathrm{H}, J=2.5 \mathrm{~Hz}$, pyrimidine), 7.10-7.81 (m, 7H, Ar-H), $8.9\left(\mathrm{~s}, 1 \mathrm{H}, \mathrm{NH}, \mathrm{D}_{2} \mathrm{O}\right.$ exchangeable), 11.5 (s, 1H, NH, $\mathrm{D}_{2} \mathrm{O}$ exchangeable); $\mathrm{Ms} \mathrm{m} / \mathrm{z}(\%): 370\left(\mathrm{M}^{+}, 33\right)$, $372\left(\mathrm{M}^{+}+2\right.$, 32). Analysis for $\mathrm{C}_{16} \mathrm{H}_{11} \mathrm{Cl}_{3} \mathrm{~N}_{2} \mathrm{~S}$ (369.7): required $\mathrm{C}, 51.98 ; \mathrm{H}, 3.00$, $\mathrm{Cl}, 28.77$; N, 7.58, S, 8.67; found C, 51.87; H, 2.9; Cl, 28.78; N, 7.67; S, 8.68.

6-(2,4-Dichlorophenyl) -3,4-dihydro -4-(thiophen-2-yl) pyrimidine-2(1H)-thione (9c)

Yield 70\%; m.p. 119-121 ${ }^{\circ} \mathrm{C}$; IR (KBr, v, $\left.\mathrm{cm}^{-1}\right)$ : 3145-3198 (NH); 1200 $(\mathrm{C}=\mathrm{S}) ; \mathrm{Ms} \mathrm{m} / \mathrm{z}(\%): 341\left(\mathrm{M}^{+}, 46\right), 343\left(\mathrm{M}^{+}+2,32\right)$. Analysis for $\mathrm{C}_{14} \mathrm{H}_{10} \mathrm{Cl}_{2} \mathrm{~N}_{2} \mathrm{~S}_{2}$ (341.28): required $\mathrm{C}, 49.27 ; \mathrm{H}, 2.95, \mathrm{Cl}, 20.78 ; \mathrm{N}, 8.21, \mathrm{~S}, 18.79$, found $\mathrm{C}, 49.28$; $\mathrm{H}, 2.92, \mathrm{Cl}, 20.70 ; \mathrm{N}, 8.01, \mathrm{~S}, 18.71$.

3-Amino-4-(substituted) -6-(2,4- dichloro- phenyl) -3,4-dihydro -1H-pyrimidine 2- thione $(10 a, b)$

To a solution of compounds $1 \mathrm{a}$ or $1 \mathrm{~b}(0.01 \mathrm{~mol})$ in absolute ethanol $(30 \mathrm{ml})$, thiosemcarbazide $(0.91 \mathrm{~g}, 0.01 \mathrm{~mol})$ and concentrated $\mathrm{HCl}(1 \mathrm{ml})$ were added. The reaction mixture was refluxed for $3 \mathrm{hr}$, then left to cool. The solid formed was filtered off, dried and crystallized from dioxane to produce (10 a,b).

3-Amino -4-(4-methoxy-phenyl) -6-(2,4- dichloro- phenyl) -3,4-dihydro - $1 \mathrm{H}$ pyrimidine -2-thione (10a)

Yield 60\%; m.p.185-186 ${ }^{\circ} \mathrm{C}$; IR (KBr, v, cm $\left.{ }^{-1}\right)$ : $3354\left(\mathrm{NH}_{2}\right), 3118(\mathrm{NH})$; ${ }^{1} \mathrm{HNMR}$ (DMSO-d $\left.\mathrm{d}_{6}, \delta \mathrm{ppm}\right): 3.75\left(\mathrm{~s}, 3 \mathrm{H}, \mathrm{OCH}_{3}\right), 4.25\left(\mathrm{br}, 2 \mathrm{H}, \mathrm{NH}_{2}, \mathrm{D}_{2} \mathrm{O}\right.$ exchangeable), 4.52 (d, $1 \mathrm{H}, J=3.11 \mathrm{~Hz}$, pyrimidine), 5.19 (d,1H,J=2.34 Hz, pyrimidine), 7.12-8.09 (m, 7H, Ar-H), 8.56 (s, 1H, NH, $\mathrm{D}_{2} \mathrm{O}$ exchangeable); MS $\mathrm{m} / \mathrm{z}(\%): 380(\mathrm{M}+, 31), 382\left(\mathrm{M}^{+}+2,22\right)$. Analysis for $\mathrm{C}_{17} \mathrm{H}_{15} \mathrm{Cl}_{2} \mathrm{~N}_{3} \mathrm{OS}(380.29)$ : required $\mathrm{C}, 53.69 ; \mathrm{H}, 3.98 ; \mathrm{Cl}, 18.65 ; \mathrm{N}, 11.05 ; \mathrm{S}, 8.43$, found $\mathrm{C}, 53.70 ; \mathrm{H}, 3.92$; $\mathrm{Cl}, 18.55 ; \mathrm{N}, 10.91 ; \mathrm{S}, 8.32$.

3-Amino -4- (4-chloro-phenyl) -6-(2,4- dichloro- phenyl) -3,4- dihydro -1H-pyrimidine 2- thione (10b)

Yield 65\%; m.p. 173-174 ${ }^{\circ} \mathrm{C}$; IR (KBr, v, cm $\left.{ }^{-1}\right): 3350\left(\mathrm{NH}_{2}\right), 3120(\mathrm{NH})$; ${ }^{1} \mathrm{HNMR}$ (DMSO-d 6 , $\delta$ ppm): 4.20 (br, $2 \mathrm{H}, \mathrm{NH}_{2}, \mathrm{D}_{2} \mathrm{O}$ exchangeable), $4.7(\mathrm{~d}, 1 \mathrm{H}$, $J=3.1 \mathrm{~Hz}$, pyrimidine), $5.10(\mathrm{~d}, 1 \mathrm{H}, J=2.36 \mathrm{~Hz}$, pyrimidine $), 7.20-7.98(\mathrm{~m}, 7 \mathrm{H}$, Ar-H), 8.6 (s, 1H, NH, D ${ }_{2} \mathrm{O}$ exchangeable); $\mathrm{MS} \mathrm{m} / \mathrm{z}(\%): 385\left(\mathrm{M}^{+}, 100\right), 387$ $\left(\mathrm{M}^{+}+2,97\right)$. Analysis for $\mathrm{C}_{16} \mathrm{H}_{12} \mathrm{Cl}_{3} \mathrm{~N}_{3} \mathrm{~S}$ (384.71): $\mathrm{C}, 49.95 ; \mathrm{H}, 3.14 ; \mathrm{Cl}, 27.65 ; \mathrm{N}$, 10.92; S, 8.33 found: C, 49.90; H, 3.12; Cl, 27.58; N, 10.83, S, 8.21. 
3-[(4-Chloro-benzylidene)-amino]-4-(4-chlorophenyl)-6-(2,4-dichlorophenyl)3,4-dihydro-1H-pyrimidine-2-thione (11)

A solution of compound 10b (3.8 g, $0.01 \mathrm{~mol}), p$-chlorobenzaldehyde $(0.01$ mol) in $30 \mathrm{ml}$ ethanol containing $3 \mathrm{ml} \mathrm{HCl}$ was refluxed for $3 \mathrm{hr}$ and the solid formed on cooling was filtered off, dried and crystallized from dioxane to give 11. Yield 57\%; m.p 210-211 ${ }^{\circ} \mathrm{C}$; ${ }^{1} \mathrm{HNMR}$ (DMSO-d ${ }_{6}, \delta$ ppm): 4.40 (d, $1 \mathrm{H} J=3.2$ $\mathrm{Hz}$, pyrimidine), $5.3(\mathrm{~d}, 1 \mathrm{H}, J=3.4 \mathrm{~Hz}$, pyrimidine), 7.2-8.0 (m, $11 \mathrm{H}, \mathrm{Ar}-\mathrm{H}), 8.4$ (s, 1H,Chif base), 10.3 (s, 1H, NH, $\mathrm{D}_{2} \mathrm{O}$ exchangeable); $\mathrm{Ms} \mathrm{m} / \mathrm{z}(\%): 507\left(\mathrm{M}^{+}\right.$, 19), $509\left(\mathrm{M}^{+}+2,18\right)$. Analysis for $\mathrm{C}_{23} \mathrm{H}_{15} \mathrm{Cl}_{4} \mathrm{~N}_{3} \mathrm{~S}$ (507.26); required $\mathrm{C}, 54.46 ; \mathrm{H}$, 2.98; Cl, 27.96; N, 8.28; S, 6.3; found C, 54.31; H; 3.1; Cl, 27.83; N, 8.37; S, 6.18 .

3-(2-Hydroxyethylamino) -6-(2,4-dichlorophenyl) -4-(4-chlorophenyl)-3,4dihydro -1-(2-hydroxyethyl) pyrimidine-2(1H)-thione (12)

To a solution of compound $10 \mathrm{~b}(3.8 \mathrm{~g}, 0.01 \mathrm{~mol})$ in dry DMF $(20 \mathrm{ml})$, sodium hydride $(0.24 \mathrm{~g}, 0.01 \mathrm{~mol})$ was added. The reaction mixture was stirred at $70{ }^{\circ} \mathrm{C}$ for $3 \mathrm{hr}$, cooled and chloroethanol $(0.01 \mathrm{~mol})$ was added, stirred at room temperature for $5 \mathrm{hr}$. The reaction mixture was evaporated under reduced pressure and the residue was solidified with distilled water, filtered off, dried then crystallized from methanol to give compound 12. Yield 45\%; m.p.140-142 ${ }^{\circ} \mathrm{C}$; IR (KBr, $v, \mathrm{~cm}^{-1}$ ): $3425(\mathrm{OH}), 3214(\mathrm{NH}) ;{ }^{1} \mathrm{HNMR}$ (DMSO-d $6, \delta$ ppm): 1.0 $\left(\mathrm{s}, 1 \mathrm{H}, \mathrm{OH}, \mathrm{D}_{2} \mathrm{O}\right.$ exchangeable $), 1.5\left(\mathrm{~s}, 1 \mathrm{H}, \mathrm{OH}, \mathrm{D}_{2} \mathrm{O}\right.$ exchangeable $), 3.2(\mathrm{t}, 4 \mathrm{H}$, $\left.J=4.2 \mathrm{~Hz}, 2 \mathrm{CH}_{2}\right), 3.6\left(\mathrm{t}, 4 \mathrm{H}, J=5.6 \mathrm{~Hz}, 2 \mathrm{CH}_{2}\right), 4.09\left(\mathrm{~s}, 1 \mathrm{H}, \mathrm{NH}, \mathrm{D}_{2} \mathrm{O}\right.$ exchangeable), $4.8(\mathrm{~d}, 1 \mathrm{H}, J=3.06 \mathrm{~Hz}$, pyrimidine), $5.4(\mathrm{~d}, 1 \mathrm{H}, J=2.09 \mathrm{~Hz}$, pyrimidine), 7.1-8.0 (m, 7H, Ar-H); $\mathrm{MS} \mathrm{m} / \mathrm{z}(\%)$ : $473\left(\mathrm{M}^{+}, 15\right), 475\left(\mathrm{M}^{+}+2,14\right)$. Analysis for $\mathrm{C}_{20} \mathrm{H}_{20} \mathrm{Cl}_{3} \mathrm{~N}_{3} \mathrm{O}_{2} \mathrm{~S}$ (472.82); required C, 50.80; H, 4.26; Cl, 22.49; N, 8.89; S, 6.78; found C, 50.75; H, 4.20; Cl, 22.36; N, 8.69; S, 6.68.

6-(2,4-Dichlorophenyl) -1,4- dihydro-4- (4-methoxyphenyl)-2-(methylthio) pyrimidine (13)

To a warmed ethanolic potassium hydroxide solution $(50 \mathrm{ml}, 0.01 \mathrm{~mol})$, compound 9a $(3.6 \mathrm{~g}, 0.01 \mathrm{~mol})$ was added for $30 \mathrm{~min}$ and the mixture was allowed to cool to room temperature. Methyl iodide $(0.012 \mathrm{~mol})$ was added to the reaction mixture with stirring for $8 \mathrm{hr}$ at $70{ }^{\circ} \mathrm{C}$. After cooling the reaction mixture was poured onto water $(100 \mathrm{ml})$, the obtained solid product was filtered off, washed with distilled water, dried and crystallized from methanol to give compound 13. Yield 70\%, m.p 160-162 ${ }^{\circ} \mathrm{C}$, IR (KBr, v, $\left.\mathrm{cm}^{-1}\right)$ : 3220 (NH); ${ }^{1} \mathrm{HNMR}\left(\mathrm{DMSO}_{\mathrm{d}}, \delta \mathrm{ppm}\right): 2.6\left(\mathrm{~s}, 3 \mathrm{H}, \mathrm{CH}_{3}\right), 3.8\left(\mathrm{~s}, 1 \mathrm{H}, \mathrm{OCH}_{3}\right), 4.1(\mathrm{~d}, 1 \mathrm{H}$, $J=2.19 \mathrm{~Hz}$, pyrimidine $\mathrm{H}), 5.7(\mathrm{~d}, 1 \mathrm{H}, J=2.29 \mathrm{~Hz}$, pyrimidine $\mathrm{H}), 6.92-8.10(\mathrm{~m}$, $7 \mathrm{H}, \mathrm{Ar}-\mathrm{H}), 10.7$ (s, 1H, NH, D $2 \mathrm{O}$ exchangeable); MS m/z (\%): $379\left(\mathrm{M}^{+}, 100\right)$ $381\left(\mathrm{M}^{+}+2,71\right)$. Analysis for $\mathrm{C}_{18} \mathrm{H}_{16} \mathrm{Cl}_{2} \mathrm{~N}_{2} \mathrm{OS}$ (379.3); required C, 57.00; H, 4.25; Cl, 18.69; S, 8.45, found; C, 57.03; H, 4.16; Cl, 18.49; S, 8.22.

Ethyl-2-(6-(2,4-dichlorophenyl) -1,4-dihydro -4-(4-methoxyphenyl) pyrimidin-2ylthio) acetate (14)

A mixture of compound 9a (3.6, 0.01mole) and ethyl chloroacetate $(1.23 \mathrm{~g}$, $0.01 \mathrm{~mol})$ in alcoholic potassium hydroxide $(30 \mathrm{ml}, 2 \%)$ was refluxed for $2 \mathrm{hr}$. Egypt. J. Chem. 55, No.4 (2012) 
The reaction mixture was poured onto acidified water, the solid formed was filtered off, dried and crystallized form ethanol to give compound 14. Yield 84\%; m.p. $148-149{ }^{\circ} \mathrm{C}$; IR $\left(\mathrm{KBr}, v, \mathrm{~cm}^{-1}\right)$ : $1735(\mathrm{C}=\mathrm{O}), 3189(\mathrm{NH}) ;{ }^{1} \mathrm{HNMR}$ (DMSO$\left.\mathrm{d}_{6}, \delta \mathrm{ppm}\right): 1.22\left(\mathrm{t}, 3 \mathrm{H}, \mathrm{CH}_{3}\right), 3.71\left(\mathrm{~s}, 3 \mathrm{H}, \mathrm{OCH}_{3}\right), 4.15\left(\mathrm{q}, 2 \mathrm{H}, \mathrm{CH}_{2}\right), 4.80(\mathrm{~s}, 2 \mathrm{H}$, $\left.\mathrm{CH}_{2}\right), 4.93(\mathrm{~d}, 1 \mathrm{H}, J=3.2 \mathrm{~Hz}$, pyrimidine $), 5.6(\mathrm{~d}, 1 \mathrm{H}, J=2.6 \mathrm{~Hz}$, pyrimidine $)$, 7.12-8.20 (m, 7H, Ar-H), 9.30 (s, 1H, NH, $\mathrm{D}_{2} \mathrm{O}$ exchangeable); $\mathrm{MS} \mathrm{m} / \mathrm{z}(\%): 451$ $\left(\mathrm{M}^{+}, 100\right), 453\left(\mathrm{M}^{+}+2,70\right)$. Analysis for $\mathrm{C}_{21} \mathrm{H}_{20} \mathrm{Cl}_{2} \mathrm{~N}_{2} \mathrm{O}_{3} \mathrm{~S}$ (451.37): required $\mathrm{C}$, $55.88 ; \mathrm{H}, 4.47 ; \mathrm{Cl}, 15.71 ; \mathrm{N}, 6.21 ; \mathrm{S}, 7.10$, found $\mathrm{C}, 55.81 ; \mathrm{H}, 4.43 ; \mathrm{Cl}, 15.60 ; \mathrm{N}$, $6.11 ; \mathrm{S}, 7.00$.

2-(4-Methoxybenzylidine) -7-(2,4-dichlorophenyl) -5-(4-methoxyphenyl) -2Hthiazolo [3,2-a]pyrimidin-3(5H)-one (15)

A mixture of compound $9 \mathrm{a}(3.6 \mathrm{~g}, 0.01 \mathrm{~mol})$, bromoacetic acid $(0.01 \mathrm{~mol})$ in acetic acid $(30 \mathrm{ml})$, acetic anhydride $(10 \mathrm{ml})$ mixture in the presence of fused anhydrous sodium acetate $(2 \mathrm{~g})$ was refluxed for $3 \mathrm{hr}$. The reaction mixture was cooled, gradually poured onto cold water, cool and filtered off, and crystallized from the benzene to give compound (15). Yield 40\%; m.p. $219-221{ }^{\circ} \mathrm{C}$; IR (KBr, $\left.v, \mathrm{~cm}^{-1}\right): 1702(\mathrm{C}=\mathrm{O}) ;{ }^{1} \mathrm{HNMR}\left(\mathrm{DMSO}_{6}, \delta \mathrm{ppm}\right): 4.95(\mathrm{~d}, 1 \mathrm{H}, J=3.1 \mathrm{~Hz}$, pyrimidine), 5.9 (d, 1H, J=3.2 Hz, pyrimidine), 7.0-8.28 (m, 10H, Ar-H), $8.8(\mathrm{~s}$, $1 \mathrm{H}, \mathrm{CH}$, methylene); MS m/z (\%):523 (M+, 100), $525\left(\mathrm{M}^{+}+2,72\right)$. Analysis for $\mathrm{C}_{27} \mathrm{H}_{20} \mathrm{Cl}_{2} \mathrm{~N}_{2} \mathrm{O}_{3} \mathrm{~S}$ (523.43): required C, 61.95; H, 3.85; Cl, 13.55; N, 5.35; S, 6.13, found $\mathrm{C}, 61.91 ; \mathrm{H}, 3.79 ; \mathrm{Cl}, 13.45 ; \mathrm{N}, 5.15 ; \mathrm{S}, 6.01$.

\section{Antioxidant activity \\ DPPH method}

The 2,2 diphenyl-1-picrylhydrazyl (DPPH) tests were carried out as described by Burits and Bucar ${ }^{(22)}$. One $\mathrm{ml}$ of synthetic compounds $(50-500 \mu \mathrm{g} / \mathrm{ml})$ was mixed with $1 \mathrm{ml}$ DPPH reagent $(0.002 \%$ (w/v) /methanol solution). After an incubation in the dark at room temperature for $30 \mathrm{~min}$, the absorbance was measured at $517 \mathrm{~nm}$ (using Jenway 6130 spectrophotometer). Silymarin (100 $\mu \mathrm{g} / \mathrm{ml}$ ) was used as positive control and extract concentration providing $50 \%$ inhibition $\left(\mathrm{IC}_{50}\right)$ was calculated from the graph plotting inhibition percentages against extract concentration. This test was carried out in triplicate and the antioxidant activity was calculated as the following:

$$
\text { Activity } \%=\text { Ac-At } / \text { Ac x } 100
$$

where: At was the absorbance of samples and Ac the absorbance of methanolic DPPH solution.

\section{Molecular modeling experimental}

All computational data were performed on Intel (R) Core (TM) 2 DUO CPU, 2.6 GHZ, $2.27 \mathrm{GHZ}$, with memory $(\mathrm{RAM})=4.00 \mathrm{~GB}$ and has Ubunto 8.04 release software. Docking was done by Plants software ${ }^{(23)}$ and autodock vina ${ }^{(24,25)}$.

All compounds were saved as pdbqt format and docking with Autodock Vina was performed according to the specified condition in which the grid box was 
adjusted to have center $\mathrm{x}=24.15$, center_y $=22.8$ and center_z $=12.9$. By these centres the pocket with the main residues were involved inside the box. Autodock vina allows the flexible docking of ligands into its site of action. It has the ability to use all the rotable bonds of the ligands to give a number of conformations from which the best mode could be achieved.

\section{References}

1. Mishra, R. and Tomar, I., International J. Pharm. Sci. Res. 2, 758 (2011).

2. Spare, N., Bahti, T., Gupta, S., Pancholi, N., Raghuvanshi, U., Dubey D., Rajopadhyay, V. and Sapre, N., J. Biophysical Chemistry, 2 , 361 (2011).

3. Rotili, D., Tarantino, D., Artico, M., Nawrozkij, M., Gonzalez-Ortega, E., Clotet, B., Samuele,A., Este, J., Maga, G. and Mai, A., J. Med. Chem. 54, 3091(2011).

4. Dudhea, R., Kumar, S., Vermae, P. and Chaudhar, A., J. Adv. Sci. Res. 2, 10 (2011).

5. Rashad, A., Mahmoud, A. and Ali, M., Eur. J. Med. Chem. 46, 1019 (2011).

6. Sayed, H. and Ali, M., Phosphrous, Sulfer and Silicon 183, 156 (2008).

7. El-Subbagh, H., Abu-Zaid, S., Mahran, M., Badria, F. and Al-Obaid, A., J. Med. Chem. 43, 2915 (2000).

8. Abd El-Ghaffar, N., Kassab, R. and Soliman, F., ChemInform, 33,157(2002).

9. Gad-Elkareem, M., Abdel-Fattah, A. and Elneairy, M., J. Sulfur Chem. 32, 273(2011).

10. Sayed, H., Abbas, H., Morsy, E. and Flefel, E., Der Pharma Chemica. 3, 31(2011).

11. Rashad, A., Shamroukh, A., Sayed, H., Awad, S. and Abdelwahed, N., Synthetic Communications, 41, 652(2011).

12. Hussein, M., Omar, R. and Farghaly, H., Inter. J. Acad. Res. 3, 454(2011).

13. Sondhi, S., Singh, N., Johar, M. and Kumar, A., Bioorg. Med. Chem. 13, 6158 (2005).

14. Mistry, P., Kamdar, N., Haveliwala, D. and Patel S., Letters in Drug Design \& Discovery, 8, 750(2011).

15. Flefel, E., Salama, M., El-Shahat, M., El-Hashash, M. and El-Farargy, A., Phosphorus, Sulfur and Silicon, 182, 1739(2007).

16. Kashyap, S., Sharma, P., Garg, V., Dudhe, R. and Kumar, N., J. Adv. Sci. Res. 2, 18(2011).

Egypt. J. Chem. 55, No.4 (2012) 
17. Beck, P., Curry, M., Chorvat, R., Fitzgerald, L. and Gilligan, P., Bioorg. Med. Chem. Lett. 9, 1185 (1999).

18. Padmaja, A., Payani, T., Reddy, G. and Padmavathi, V., European Journal of Medicinal Chemistry, 44, 4557(2009).

19. Harman, D., J. Am. Geriatr. Soc. 20, 145(1972).

20. Sun, L. and McMahon, G., Drug Discovery Today, 5, 344 (2000).

21. John, R. and Glenney, J.R., Biochimica et Biophysica Acta (BBA) - Molecular Cell Research, 1134, 113(1992).

22. Burits, M. and Bucar, F., Phytother Res. 14, 323 (2000).

23. Korb, O. and Stutzle, E., Swarm Intelligence, 1, 115(2007).

24. Trott, O., Olson, J. and Vina, A., Journal of Computational Chemistry, 31, 455(2010).

25. Michel, F. and Python S., J. Mol. Graphics and Mod, 17, 57(1999).

(Received 7/10/2012;

accepted 4/11/2012) 


\title{
التحضيروالالتحام الجزيئى وتقييم النشاط البيولوجى لبعض \\ المركبات الحلقية الجديدة
}

\author{
إيمان محمد فليفل ، وليد السيد طنطاوى ، أحمد الفايد ، هيام حسنين سيا ومحمد \\ قسم الكيمياء الضوئيةــ المركز القومى للبحوث و * قسم الكيمياء الصيدليةـ كلية \\ الصيدلة - جامعة حلوان- مصر. الصني
}

\begin{abstract}
نظراً لأهمية المشتقات الحلقية الغير متجانسة الحلقة والمحتوية على أنوية البيريميدينون والبريميدينثيون من الناحية الفارماكولوجية والطبية حيثة الطية أنها تدخل فى صناعة الأدوية المختلفة وأيضا من الناحية البيولوجية ولفية والتطبيقات

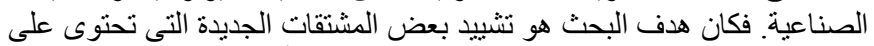

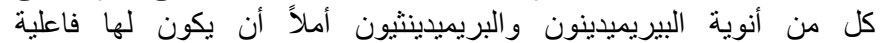

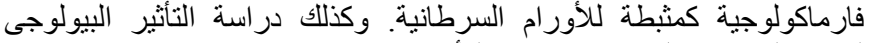
لبعض المركبات الناتجة كمضادات للأكسدة.
\end{abstract}

تم تحضير سلسلة من المشتقات الحلقية غير المتجانسة تشمل البريميدينون

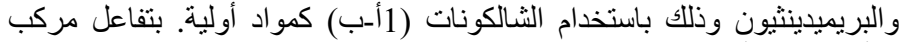
(1أ) مع فوق أكسيد الهيدروجين ينتج مركب اييوكسيد (2) الذي يتفاعل مع الثيوريا

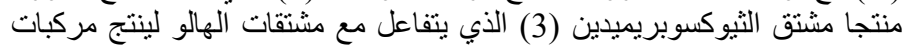

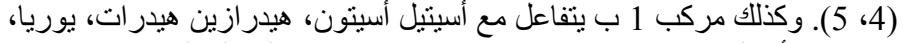

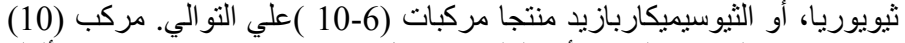

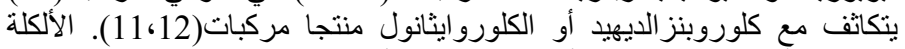

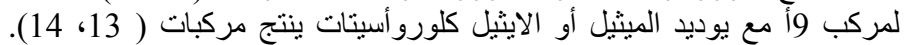

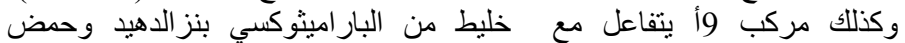
البروموخليك للحصول علي ثيازولوبريميدين ( 15) (مخططات 2، 1، 1).

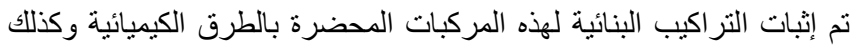

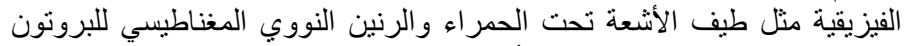

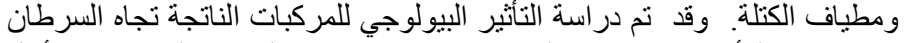

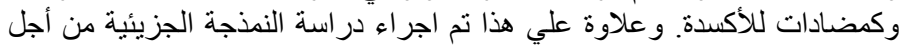

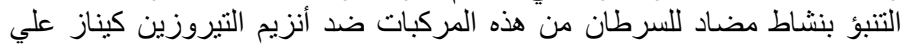

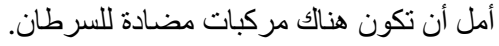

